\title{
A CONFIGURATION OF LINES IN THREE DIMENSIONS
}

\author{
by J. W. P. HIRSCHFELD
}

(Received 30th July 1971)

\section{Introduction}

In 1849, Cayley and Salmon discovered that a general cubic surface in projective space of three dimensions over the complex numbers has twentyseven lines on it. They remarked that all the properties of the twenty-seven lines would not become apparent until a better notation than they had given was found. This notation was discovered by Schläfli in 1858 in the double-six

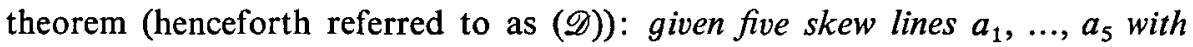
a single transversal $b_{6}$ such that no four of the $a_{i}$ lie in a regulus, the four $a_{i}$ excluding $a_{j}$ have a second transversal $b_{j}$ and the five lines $b_{1}, \ldots, b_{5}$ thus obtained have a transversal $a_{6}$-the completing line of the double-six. The other fifteen lines of the cubic surface are then $c_{i j}=a_{i} b_{j} \cap a_{j} b_{i}$, where $a_{i} b_{j}$ is the plane containing $a_{i}$ and $b_{j}$.

In 1898, Grace gave the following extension of the double-six theorem: given six skew lines $c_{1}, \ldots, c_{6}$ with a transversal $\alpha$ such that no four of the $c_{i}$ lie in a regulus and no five have a further transversal, then $\alpha$ and the set of five $c_{i}$ excluding $c_{j}$ determine a double-six with completing line $d_{j}$ and the six lines $d_{1}, \ldots, d_{6}$ have a transversal $\beta$ - the Grace line.

It is natural to ask whether Grace's theorem may be similarly extended and whether there is an infinite sequence of such theorems. Thus the first question is: if, from seven skew lines $A_{1}, \ldots, A_{7}$ with a transversal $\Delta$, the set of six $A_{i}$ excluding $A_{j}$ determine the Grace line $\Gamma_{j}$, do the seven Grace lines $\Gamma_{1}, \ldots, \Gamma_{7}$ have a transversal?

In (9), this question was answered in the negative by the following method. If the theorem is true over the complex field, say, the lines $\Gamma_{1}, \ldots, \Gamma_{7}$ can be obtained from the lines $A_{1}, \ldots, A_{7}$ by solving certain sets of linear equations. If the line coordinates of $A_{i}$ are $A_{i}^{(1)}, \ldots, A_{i}^{(6)}$, then the line coordinates of $\Gamma_{1}, \ldots, \Gamma_{7}$ will lie in the polynomial ring $Z\left[\left\{A_{i}^{(j)}\right\}\right]$, where $Z$ is the ring of integers. Let $\Gamma_{1}, \ldots, \Gamma_{5}$ lie in the linear complex $W$. Then $\Gamma_{1}, \ldots, \Gamma_{7}$ have a transversal if $W$ is special and if both $\Gamma_{6}$ and $\Gamma_{7}$ lie in $W$. Thus three identities must be satisfied in the ring $Z\left[\left\{A_{i}^{(j)}\right\}\right]$. Therefore, if the theorem is true over the complex field, the theorem is true over any field for which the lines $\Gamma_{1}, \ldots, \Gamma_{7}$ exist and which is a homomorphic image of $Z$. In (9), the field $G F(31)$ was chosen and, with the aid of a computer, a set of $A_{1}, \ldots, A_{7}$ found which produced the lines $\Gamma_{1}, \ldots, \Gamma_{7}$. They did not have a transversal. So the question E.M.S. - G 
was negatively answered. The same result has since been found independently by Longuet-Higgins (11), who gave two examples over the real field. In both cases, the seven lines $\Gamma_{1}, \ldots, \Gamma_{7}$ belonged to a (non-special) linear complex, confirming in these cases a conjecture due to Babbage.

Two questions now arise. Firstly, can this result be proved without using a computer? Secondly, what is the complete configuration obtained from $\Delta$ and $A_{1}, \ldots, A_{7}$ ? This paper answers the second question.

Grace (6) originally proved his theorem by considering six hyperspheres through a point in four dimensions. Every four have a second point in common. Thus each set of five hyperspheres produces five points. These five points lie on a hypersphere. So, from six hyperspheres, six sets of five can be formed and from each set of five a new hypersphere is obtained. It was shown that the six new hyperspheres have a point in common.

This result was transformed to one on lines and linear complexes in three dimensions, and this result in turn was specialised by using special linear complexes and by involving $(\mathscr{D})$ to give the required result.

Brown (3) considered an extension of Grace's theorem in four dimensions by commencing with seven hyperplanes through a point. This transforms into a theorem in three dimensions about seven linear complexes with a line in common, but says nothing about seven lines with a transversal. Nevertheless, we shall show that the analogy does hold. The review by Coxeter (4) of Brown's paper was extremely helpful in suggesting a revised notation similar to that below, as well as in identifying the group of the configuration as a familiar one.

\section{Notation and preliminaries}

The geometry throughout takes place in a projective space of three dimensions over an arbitrary field $K$ with the single condition that $K$ is large enough for the configuration to exist: it was shown in (7) and (8) that the double-six exists for all $K$ except $G F(q)$ with $q=2,3$ or 5 and that, for Grace's figure to exist, $q \geqq 9$.

Indices written on the same level will be interchangeable; e.g. $\Gamma_{j k l}^{i}=\Gamma_{p q r}^{i}$ where $p q r$ is any permutation of $j k l$.

$\mathscr{R}\left(l_{1} l_{2} \ldots l_{n}\right)$ indicates that the lines $l_{1}, l_{2}, \ldots, l_{n}$ lie in a regulus.

To establish the configuration, two theorems will be required-(OD) and Kubota's theorem (henceforth referred to as $(\mathscr{K})$ ). Grace's theorem was proved by Wren (14) in the following way: beginning with the lines $c_{1}, \ldots, c_{6}$ with transversal $\alpha$, the four $c_{i}$ excluding $c_{j}$ and $c_{k}$ have a further transversal $\alpha_{j k}$ and the five lines $\alpha_{i j}$ with $j=1, \ldots, 6, j \neq i$, have a transversal $d_{i}$ by (D). It was then shown that the six lines $c_{1}, c_{2}, d_{3}, d_{4}, d_{5}, d_{6}$ have a transversal $\beta_{12}$. By constructing double-sixes from the lines $d_{i}$ and $\beta_{i j}$ similar to those with the lines $c_{i}$ and $\alpha_{i j}$, it was shown that the six lines $d_{i}$ have a transversal $\beta$. The existence of the lines $\beta_{i j}$ was shown differently by Kubota (10) as follows. In 


\section{A CONFIGURATION OF LINES IN THREE DIMENSIONS}

the above situation, the four reguli $\left(\alpha_{12} \alpha_{13} \alpha_{14}\right),\left(\alpha_{12} \alpha_{23} \alpha_{24}\right),\left(\alpha_{13} \alpha_{23} \alpha_{34}\right)$, $\left(\alpha_{14}, \alpha_{24}, \alpha_{34}\right)$ have a common line $\beta_{56}$ : this theorem is $(\mathscr{K})$. By mapping the lines on to the Klein quadric in five dimensions and projecting on to a plane, it is equivalent to the result that the four circumcircles of the four triangles obtained by omitting in turn each of four lines in the plane have a common point (8).

The configuration to be constructed consists of two types of lines, which will be called Greek (g-lines) and Latin (1-lines) and denoted accordingly. The first table below gives in stages the list of results either assumed or to be proved in $\S 3$. The second table gives the intersections between the g-lines and the I-lines as they are obtained. Here, the right-hand column lists the number of l-lines that the given g-line meets. The third table lists the numbers of 1-lines and g-lines. In all, the g-lines and 1-lines form a tactical configuration $(576,7 ; 56,72)$, in which each of the 576 g-lines meets seven l-lines and each of the 56 l-lines meets $72 \mathrm{~g}$-lines.

\section{Stages of the Configuration}

$S_{1}: \quad A_{1}, A_{2}, A_{3}, A_{4}, A_{5}, A_{6}, A_{7}$ have the transversal $\Delta$

$S_{2}: \quad A_{1}, A_{2}, A_{3}, A_{4}$ also have the transversal $\Delta_{567}$

$S_{3}: \quad \Delta_{123}, \Delta_{124}, \Delta_{125}, \Delta_{126}, \Delta_{127}$ have the transversal $A_{12}$

$S_{4}: \quad A_{12}, A_{13}, A_{14}, A_{15}, A_{6}, A_{7}$ have the transversal $\Gamma_{67}^{1}$

$S_{5}: \quad A_{12}, A_{13}, A_{14}, A_{15}, A_{16}, A_{17}$ have the transversal $\Gamma_{1}$

$S_{6}: \quad A_{1}, A_{56}, A_{57}, A_{67}$ have the transversals $\Delta_{567}$ and $\Delta_{567}^{1}$

$S_{7}: \quad \Delta_{i j k}^{1}, \Delta_{i j k}^{2}(\forall i, j, k \neq 1,2)$ have the transversal $B_{12}$

$S_{8}: \quad A_{12}, A_{13}, A_{14}, B_{56}, B_{57}, B_{67}$ have the transversal $\Gamma_{567}^{1}$

$S_{9}: A_{1}, B_{12}, B_{13}, B_{14}, B_{15}, B_{16}, B_{17}$ have the transversal $\Delta_{1}$

$S_{10}: A_{23}, B_{14}, B_{15}, B_{16}, B_{17}$ have the transversal $\Delta_{23}^{1}$

$S_{11}: \Gamma_{i j k}^{1}(\forall i, j, k \neq 1)$ has the transversal $B_{1}$

$S_{12}: \quad B_{1}$ meets $\Gamma_{1}$

$S_{13}: \quad B_{1}, B_{2}, B_{3}, B_{4}, B_{56}, B_{57}, B_{67}$ have the transversal $\Gamma_{567}$

$S_{14}: B_{1}, B_{2}, B_{3}, B_{4}, B_{5}, B_{6}, B_{7}$ have the transversal $\Gamma$ 


\section{Intersections of the Configuration}

$\begin{array}{lllll}S_{1}: & \Delta & \text { meets } & A_{i} & 7 \\ S_{2}, S_{3}: & \Delta_{i j k} & \text { meets } & A_{l}, A_{i j} & 7 \\ S_{4}: & \Gamma_{j k}^{i} & \text { meets } & A_{j}, A_{i l} & 6 \\ S_{5}: & \Gamma_{i} & \text { meets } & A_{i j} & 6 \\ S_{6}: & \Delta_{j k l}^{i} & \text { meets } & A_{i}, A_{j k} & 4 \\ S_{7}: & \Gamma_{j k}^{i} & \text { meets } & B_{j k} & 1 \\ S_{7}: & \Delta_{j k l}^{i} & \text { meets } & B_{i m} & 3 \\ S_{8}: & \Gamma_{j k l}^{i} & \text { meets } & A_{i m}, B_{j k} & 6 \\ S_{9}: & \Delta_{i} & \text { meets } & A_{i}, B_{i j} & 7 \\ S_{10}: & \Delta_{j k}^{i} & \text { meets } & A_{j k}, B_{i l} & 5 \\ S_{11}: & \Gamma_{j k l}^{i} & \text { meets } & B_{i} & 1 \\ S_{11}: & \Delta_{j k}^{i} & \text { meets } & B_{j} & 2 \\ S_{12}: & \Gamma_{i} & \text { meets } & B_{i} & 1 \\ S_{13}: & \Gamma_{i j k} & \text { meets } & B_{l}, B_{i j} & 7 \\ S_{14}: & \Gamma & \text { meets } & B_{i} & 7\end{array}$

\section{Lines of the Configuration}

Latin

Greek

\begin{tabular}{|c|c|c|}
\hline & & $\Delta$ \\
\hline 7 & $A_{i}$ & $\Delta_{i j k}$ \\
\hline 21 & $A_{i j}$ & $\Gamma_{j k}^{i}$ \\
\hline & & $\Gamma_{i}$ \\
\hline & & $\Delta_{j k l}^{i}$ \\
\hline 21 & $B_{i j}$ & $\Gamma_{j k l}^{i}$ \\
\hline & & $\Delta_{i}$ \\
\hline 7 & $B$ & $\Delta_{j k}^{i}$ \\
\hline & & $\Gamma_{i j k}$ \\
\hline & & $\Gamma$ \\
\hline
\end{tabular}




\section{A CONFIGURATION OF LINES IN THREE DIMENSIONS 109}

To set the picture, there follow some elementary results on line geometry.

(1) Four skew lines lie in a regulus if and only if they are linearly dependent.

(2) Four skew lines have only one transversal if and only if the four skew lines are linearly independent and the five lines are linearly dependent.

(3) Five skew lines, four of which have just two transversals, have two transversals if and only if they are linearly dependent.

(4) Six skew lines lie in a linear complex if and only if they are linearly dependent.

(5) Five skew lines have exactly one transversal if and only if the six lines are linearly dependent but the five are not.

(6) Five skew lines have exactly one transversal and are part of a doublesix if and only if the six are linearly dependent but no five of the six are.

(7) A necessary condition that six skew lines with a transversal lead to Grace's theorem is that every five of the seven lines are linearly independent (this condition is not quite sufficient (8), p. 357).

(8) A necessary condition that seven skew lines with a transversal lead to Brown's configuration is that every five of the eight lines are linearly independent.

\section{Construction of the configuration}

$S_{1}$. Let us begin with the seven lines $A_{i}$ with transversal $\Delta$ satisfying condition (8) above.

$S_{2} . A_{1}, A_{2}, A_{3}, A_{4}$ have the further transversal $\Delta_{567}$.

$S_{3}$. By $(\mathscr{D}), \Delta_{123}, \Delta_{124}, \Delta_{125}, \Delta_{126}, \Delta_{127}$ have a transversal $A_{12}$. Thus there are 21 double-sixes like

$$
\begin{array}{llllll}
A_{12} & A_{3} & A_{4} & A_{5} & A_{6} & A_{7} \\
\Delta & \Delta_{123} & \Delta_{124} & \Delta_{125} & \Delta_{126} & \Delta_{127} .
\end{array}
$$

$S_{4}$. Apply $(\mathscr{K})$ to the case $c_{1}=A_{2}, c_{2}=A_{3}, c_{3}=A_{4}, c_{4}=A_{5}, c_{5}=A_{6}$, $c_{6}=A_{7}$. Then $\alpha_{12}=\Delta_{123}, \alpha_{13}=\Delta_{124}, \alpha_{14}=\Delta_{125}, \alpha_{23}=\Delta_{134}, \alpha_{24}=\Delta_{135}$, $\alpha_{34}=\Delta_{145}$. So the reguli in the left-hand column below have a common line: their complementary reguli are written on the right.

$\begin{array}{lllllll}\Delta_{123} & \Delta_{124} & \Delta_{125} & A_{6} & A_{7} & A_{12} \\ \Delta_{132} & \Delta_{134} & \Delta_{135} & & A_{6} & A_{7} & A_{13} \\ \Delta_{142} & \Delta_{143} & \Delta_{145} & & A_{6} & A_{7} & A_{14} \\ \Delta_{152} & \Delta_{153} & \Delta_{154} & & A_{6} & A_{7} & A_{15}\end{array}$

Let this common line be $\Gamma_{67}^{1}$; it therefore meets $A_{12}, A_{13}, A_{14}, A_{15}, A_{6}, A_{7}$.

$S_{5}$. Theorem. All fifteen lines $\Gamma_{i j}^{1}$ are distinct. 


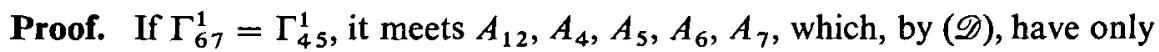
the one transversal $\Delta_{123}$; so $\Gamma_{67}^{1}=\Delta_{123}$. Then, by $(\mathscr{K})$, we have

$$
\mathscr{R}\left(\Delta_{123} \Delta_{142} \Delta_{143} \Delta_{145}\right)
$$

which implies $\mathscr{R}\left(A_{14} A_{5} A_{6} A_{7}\right)$, contradicting the existence of a double-six such as the above. So $\Gamma_{67}^{1} \neq \Gamma_{45}^{1}$.

If $\Gamma_{67}^{1}{ }^{1}=\Gamma_{57}^{1}$, it meets $A_{5}, A_{6}, A_{7}, A_{12}, A_{13}, A_{14}$. But the three sets of four $\left\{A_{5}, A_{6}, A_{7}, A_{12}\right\},\left\{A_{5}, A_{6}, A_{7}, A_{13}\right\},\left\{A_{5}, A_{6}, A_{7}, A_{14}\right\}$ have just the respective pairs of transversals $\left\{\Delta_{123}, \Delta_{124}\right\},\left\{\Delta_{123}, \Delta_{134}\right\},\left\{\Delta_{124}, \Delta_{134}\right\}$. As all $\Delta_{i j k}$ are distinct, the six lines have no transversal. So $\Gamma_{67}^{1} \neq \Gamma_{57}^{1}$. So all fifteen lines $\Gamma_{i j}^{1}$ are distinct.

At this stage, in order that the figure does not degenerate, it must be assumed that any set of six lines like $A_{12}, A_{13}, A_{14}, A_{15}, A_{16}, A_{17}$ are skew: in fact, the six lines are all skew or all concurrent, (8), p. 354.

It was previously shown that the four lines $A_{5}, A_{6}, A_{7}, A_{12}$ do not lie in a regulus.

Theorem. No four of the lines $A_{6}, A_{7}, A_{12}, A_{13}, A_{14}, A_{15}$ lie in a regulus.

Proof. $\mathscr{R}\left(A_{6}, A_{7}, A_{12}, A_{13}\right) \Rightarrow \mathscr{R}\left(\Gamma_{67}^{1}, \Delta_{123}, \Delta_{124}, \Delta_{134}\right)$

$$
\begin{aligned}
& \Rightarrow \mathscr{R}\left(A_{5}, A_{6}, A_{7}, A_{12}\right) . \\
\mathscr{R}\left(A_{7}, A_{12}, A_{13}, A_{14}\right) & \Rightarrow \mathscr{R}\left(\Gamma_{67}^{1}, \Delta_{123}, \Delta_{124}, \Delta_{134}\right) . \\
\mathscr{R}\left(A_{12}, A_{13}, A_{14}, A_{15}\right) & \Rightarrow \mathscr{R}\left(\Gamma_{26}^{1}, \Gamma_{27}^{1}, \Gamma_{36}^{1}, \Gamma_{67}^{1}\right) \\
& \Rightarrow \mathscr{R}\left(A_{6}, A_{7}, A_{12}, A_{13}\right) .
\end{aligned}
$$

So, in all cases, there is a contradiction.

There are $7 \times\left(\begin{array}{l}6 \\ 3\end{array}\right)=140$ double-sixes of the type

$$
\begin{array}{llllll}
A_{2} & A_{3} & A_{4} & A_{15} & A_{16} & A_{17} \\
\Gamma_{34}^{1} & \Gamma_{24}^{1} & \Gamma_{23}^{1} & \Delta_{167} & \Delta_{157} & \Delta_{156}
\end{array}
$$

and double-sixes such as

and

$$
\begin{array}{llllll}
A_{2} & A_{13} & A_{14} & A_{15} & A_{16} & A_{17} \\
\Gamma_{1} & \Gamma_{23}^{1} & \Gamma_{24}^{1} & \Gamma_{25}^{1} & \Gamma_{26}^{1} & \Gamma_{27}^{1}
\end{array}
$$

$$
\begin{array}{cccccc}
A_{3} & A_{12} & A_{14} & A_{15} & A_{16} & A_{17} \\
\Gamma_{1}^{*} & \Gamma_{23}^{1} & \Gamma_{34}^{1} & \Gamma_{35}^{1} & \Gamma_{36}^{1} & \Gamma_{37}^{1} .
\end{array}
$$

Theorem. $\Gamma_{1}^{*}=\Gamma_{1}$.

Proof. As $A_{14}, A_{15}, A_{16}, A_{17}$ lie in one half of a double-six and meet $\Gamma_{23}^{1}$, they have just one further transversal. So $\Gamma_{1}^{*}=\Gamma_{1}$. 


\section{A CONFIGURATION OF LINES IN THREE DIMENSIONS 111}

There are then 42 double-sixes of the above type.

The lines $\Gamma_{i}, i=1, \ldots, 7$, are the Grace lines.

$S_{6} . A_{1}, A_{56}, A_{57}, A_{67}$ have the transversal $\Delta_{567}$. The four lines do not lie in a regulus, since

$$
\begin{aligned}
\mathscr{R}\left(A_{1} A_{56} A_{57} A_{67}\right) & \Rightarrow \mathscr{R}\left(\Gamma_{12}^{5} \Gamma_{13}^{5} \Gamma_{14}^{5} \Gamma_{12}^{6} \Gamma_{13}^{6} \Gamma_{14}^{6} \Gamma_{12}^{7} \Gamma_{13}^{7} \Gamma_{14}^{7} \Delta_{567}\right) \\
& \Rightarrow \mathscr{R}\left(A_{1} A_{2} A_{56} A_{57}\right),
\end{aligned}
$$

contradicting a theorem in $S_{5}$. So $A_{1}, A_{56}, A_{57}, A_{67}$ have a second transversal $\Delta_{567}^{1}$. (It is here assumed that the degeneracy $\Gamma_{12}^{6}=\Gamma_{12}^{7}$ has not occurred: if $\Gamma_{12}^{6}=\Gamma_{12}^{7}$, then this line meets $A_{1}, A_{2}, A_{36}, A_{37}$ and is $\Gamma_{12}^{3}$ or $\Delta_{367}$. If $\Gamma_{12}^{6}=\Delta_{367}$, then $\Delta_{367}$ meets $A_{46}$, which is impossible as they are polar in a double-six. Hence $\Gamma_{12}^{6}=\Gamma_{12}^{3}$ and all $\Gamma_{12}^{i}$ are the same. In general, as will be shown below, the five lines $\Gamma_{12}^{i}$ lie in a regulus.)

$S_{7}$. Now consider $\Delta_{567}$ meeting the five lines $A_{1}, A_{2}, A_{56}, A_{57}, A_{67}$. Every four have a unique second transversal. So, by $(\mathscr{D})$, we have the double-six

$$
\begin{array}{llllll}
B_{567}^{12} & A_{1} & A_{2} & A_{67} & A_{57} & A_{56} \\
\Delta_{567} & \Delta_{567}^{2} & \Delta_{567}^{1} & \Gamma_{12}^{5} & \Gamma_{12}^{6} & \Gamma_{12}^{7} .
\end{array}
$$

Theorem. The ten lines $B_{i j k}^{12}$ are identical.

Proof. Apply $(\mathscr{K})$ to the case $\alpha=\Delta_{567}, c_{1}=A_{1}, c_{2}=A_{3}, c_{3}=A_{56}$, $c_{4}=A_{57}, c_{5}=A_{2}, c_{6}=A_{67}$. Then

$$
\alpha_{12}=\Delta_{567}^{2}, \alpha_{13}=\Gamma_{23}^{7}, \alpha_{14}=\Gamma_{23}^{6}, \alpha_{23}=\Gamma_{12}^{7}, \alpha_{24}=\Gamma_{12}^{6}, \alpha_{34}=\Delta_{467} .
$$

Thus we have

$$
\begin{array}{llllll}
\Delta_{567}^{2} & \Gamma_{23}^{7} & \Gamma_{23}^{6} & A_{2} & A_{67} & B_{567}^{23} \\
\Delta_{567}^{2} & \Gamma_{12}^{7} & \Gamma_{12}^{6} & A_{2} & A_{67} & B_{567}^{12} \\
\Gamma_{23}^{7} & \Gamma_{12}^{7} & \Delta_{467} & A_{2} & A_{67} & A_{47} \\
\Gamma_{23}^{6} & \Gamma_{12}^{6} & \Delta_{467} & A_{2} & A_{67} & A_{46} .
\end{array}
$$

The left-hand reguli have a common line. The other transversal to $A_{2}, A_{67}$, $A_{47}, A_{46}$ apart from $\Delta_{467}$ is $\Delta_{467}^{2}$ : this is then the required line. So $\Delta_{467}^{2}$ meets $B_{567}^{12}$; similarly $\Delta_{467}^{1}$ meets $B_{567}^{12}$. So $B_{567}^{12}$ meets $\Delta_{467}^{1}, \Delta_{467}^{2}, \Gamma_{12}^{6}, \Gamma_{12}^{7}$.

Consider now the double-six

$$
\begin{array}{llllll}
B_{467}^{12} & A_{1} & A_{2} & A_{67} & A_{47} & A_{46} \\
\Delta_{467} & \Delta_{467}^{2} & \Delta_{467}^{1} & \Gamma_{12}^{4} & \Gamma_{12}^{6} & \Gamma_{12}^{7} .
\end{array}
$$

Therefore $\Delta_{467}^{1}, \Delta_{467}^{2}, \Gamma_{12}^{6}, \Gamma_{12}^{7}$ have the three transversals $B_{567}^{12}, B_{467}^{12}, A_{67}$, where $A_{67} \neq B_{467}^{12}$ and $A_{67} \neq B_{567}^{12}$. As the above four lines do not belong to a regulus, $B_{467}^{12}=B_{567}^{12}$. Thus all ten lines $B_{i j k}^{12}$ are identical. 
Write $B_{12}$ for $B_{i j k}^{12}$. There are $\left(\begin{array}{l}7 \\ 2\end{array}\right) \times\left(\begin{array}{l}5 \\ 3\end{array}\right)=210$ double-sixes of the form

$$
\begin{array}{llllll}
B_{12} & A_{1} & A_{2} & A_{45} & A_{35} & A_{34} \\
\Delta_{345} & \Delta_{345}^{2} & \Delta_{345}^{1} & \Gamma_{12}^{3} & \Gamma_{12}^{4} & \Gamma_{12}^{5}
\end{array}
$$

and all twenty lines $\Delta_{i j k}^{1}, \Delta_{i j k}^{2}(i, j, k \neq 1,2)$ meet $B_{12}$. Further $\mathscr{R}\left(A_{1} A_{2} B_{12}\right)$ implies $\mathscr{R}\left(\Gamma_{12}^{3} \Gamma_{12}^{4} \Gamma_{12}^{5} \Gamma_{12}^{6} \Gamma_{12}^{7}\right)$.

$S_{8}$. Now consider $A_{5}$ meeting $\Delta_{134}^{5}, \Delta_{124}^{5}, \Delta_{123}^{5}, \Gamma_{56}^{1}, \Gamma_{57}^{1}$. No four lie in a regulus since $\mathscr{R}\left(\Delta_{123}^{5} \Delta_{124}^{5} \Gamma_{56}^{1} \Gamma_{57}^{1}\right) \Rightarrow \mathscr{R}\left(A_{5} A_{12} A_{13} A_{14}\right)$ and

also.

$$
\mathscr{R}\left(\Delta_{123}^{5} \Delta_{124}^{5} \Delta_{134}^{5} \Gamma_{56}^{1}\right) \Rightarrow \mathscr{R}\left(A_{5} A_{12} A_{13} A_{14}\right)
$$

Therefore, by $(\mathscr{D})$, we have the double-sixes

and

$$
\begin{array}{llllll}
A_{12} & A_{13} & A_{14} & B_{56} & B_{57} & A_{5} \\
\Delta_{134}^{5} & \Delta_{124}^{5} & \Delta_{123}^{5} & \Gamma_{57}^{1} & \Gamma_{56}^{1} & \Gamma_{567}^{1}
\end{array}
$$

$$
\begin{array}{llllll}
A_{12} & A_{13} & A_{14} & B_{56} & B_{67} & A_{6} \\
\Delta_{134}^{6} & \Delta_{124}^{6} & \Delta_{123}^{6} & \Gamma_{67}^{1} & \Gamma_{56}^{1} & \Gamma_{567}^{1 *} .
\end{array}
$$

Theorem. $\quad \Gamma_{567}^{1 *}=\Gamma_{567}^{1}$.

Proof. As $A_{12}, A_{13}, A_{14}, B_{56}$ lie in one half of a double-six and meet $\Gamma_{56}^{1}$, they have just one further transversal. So $\Gamma_{567}^{1 *}=\Gamma_{567}^{1}$.

$\Gamma_{567}^{1}$ meets $A_{12}, A_{13}, A_{14}, B_{56}, B_{57}, B_{67}$ and there are $7 \times\left(\begin{array}{l}6 \\ 3\end{array}\right) \times 3=420$ double-sixes of the above type.

$S_{9}$. Consider the six lines $A_{1}, A_{23}, B_{14}, B_{15}, B_{16}, B_{17}$. It will ultimately be shown that they form one half of a double-six.

Theorem. No four of $A_{1}, A_{23}, B_{14}, B_{15}, B_{16}, B_{17}$ lie in a regulus.

Proof. Similarly to the one above, the following six lines form one-half of a double-six and so no four lie in a regulus: $A_{23}, A_{26}, A_{27}, B_{14}, B_{15}, A_{1}$.

$$
\mathscr{R}\left(A_{23} B_{14} B_{15} B_{16}\right) \text { implies that } \Gamma_{156}^{2} \text { meets } B_{14} \text {. }
$$

Hence

$$
\begin{aligned}
\mathscr{R}\left(\Gamma_{145}^{2} \Gamma_{156}^{2} \Delta_{237}^{1}\right) & \Rightarrow \mathscr{R}\left(A_{23} A_{27} B_{14} B_{15}\right) . \\
\mathscr{R}\left(B_{14} B_{15} B_{16} B_{17}\right) & \Rightarrow \mathscr{R}\left(\Delta_{234}^{1} \Delta_{235}^{1} \Delta_{236}^{1} \Delta_{237}^{1}\right) \\
& \Rightarrow \mathscr{R}\left(A_{1} A_{23} B_{14} B_{15}\right) . \\
\mathscr{R}\left(A_{1} B_{14} B_{15} B_{16}\right) & \Rightarrow \mathscr{R}\left(\Delta_{234}^{1} \Delta_{235}^{1} \Delta_{236}^{1} \Delta_{237}^{1}\right) .
\end{aligned}
$$

In each case, there is a contradiction. So no four of the given six lines lie in a regulus. 
$\Delta_{234}^{1}$ meets $A_{1}, A_{23}, A_{24}, A_{34}, B_{15}, B_{16}, B_{17}$. Let the second transversal of $A_{1}, B_{15}, B_{16}, B_{17}$ be $\Delta_{1}$.

Theorem. $\Delta_{1}$ meets $B_{12}, B_{13}, B_{14}$.

Proof. Apply $(\mathscr{K})$ to the case $\alpha=\Delta_{234}^{1}, c_{1}=A_{24}, c_{2}=A_{34}, c_{3}=B_{15}$, $c_{4}=B_{16}, c_{5}=B_{17}, c_{6}=A_{1}$. Then

$$
\alpha_{12}=\Delta_{1}, \alpha_{13}=\Delta_{345}^{1}, \alpha_{14}=\Delta_{346}^{1}, \alpha_{23}=\Delta_{245}^{1}, \alpha_{24}=\Delta_{246}^{1}, \alpha_{34}=\Gamma_{17}^{4} .
$$

Thus we have

$$
\begin{array}{llllll}
\Delta_{1} & \Delta_{345}^{1} & \Delta_{346}^{1} & A_{1} & B_{17} & X \\
\Delta_{1} & \Delta_{245}^{1} & \Delta_{246}^{1} & A_{1} & B_{17} & Y \\
\Delta_{345}^{1} & \Delta_{245}^{1} & \Gamma_{17}^{4} & A_{1} & B_{17} & A_{45} \\
\Delta_{346}^{1} & \Delta_{246}^{1} & \Gamma_{17}^{4} & A_{1} & B_{17} & A_{46} .
\end{array}
$$

The reguli on the left all have a line $Z$ in common, which meets all the lines on the right. But the only transversal of $A_{1}, B_{17}, A_{45}, A_{46}$ other than $\Gamma_{17}^{4}$ is $\Delta_{456}^{1}$. So $Z$ is $\Delta_{456}^{1}$. Hence $\mathscr{R}\left(\Delta_{1} \Delta_{345}^{1} \Delta_{346}^{1} \Delta_{456}^{1}\right)$. Hence $B_{12}$, which meets the last three lines meets $\Delta_{1}$. Also $\mathscr{R}\left(\Delta_{1} \Delta_{245}^{1} \Delta_{246}^{1} \Delta_{456}^{1}\right) ; B_{13}$ meets the last three lines and therefore $\Delta_{1}$. Similarly, by putting $c_{1}=A_{23}$ above, it may be shown that $B_{14}$ meets $\Delta_{1}$.

Therefore it has been shown that $A_{1}, B_{12}, B_{13}, B_{14}, B_{15}, B_{16}, B_{17}$ have the transversal $\Delta_{1}$.

$S_{10}$. Now consider $\Delta_{1}$ meeting $A_{1}, B_{14}, B_{15}, B_{16}, B_{17}$. No four of the five lines lie in a regulus. So, by $(\mathscr{D})$, the double-six may be completed:

$$
\begin{array}{llllll}
A_{23} & A_{1} & B_{14} & B_{15} & B_{16} & B_{17} \\
\Delta_{1} & \Delta_{23}^{1} & \Delta_{234}^{1} & \Delta_{235}^{1} & \Delta_{236}^{1} & \Delta_{237}^{1} .
\end{array}
$$

There are $7 \times\left(\begin{array}{l}6 \\ 2\end{array}\right)=105$ double-sixes of this type and 105 lines $\Delta_{j k}^{i}$.

$S_{11}$. Consider the construction of Grace's figure on $\Delta_{1}$ and $B_{12}, B_{13}, B_{14}$, $B_{15}, B_{16}, B_{17}$. This gives double-sixes like

$$
\begin{array}{llllll}
B_{1}^{2} & B_{13} & B_{14} & B_{15} & B_{16} & B_{17} \\
\Delta_{1} & \Delta_{23}^{1} & \Delta_{24}^{1} & \Delta_{25}^{1} & \Delta_{26}^{1} & \Delta_{27}^{1} .
\end{array}
$$

Theorem. All six lines $B_{i}^{7}(i \neq 7)$ are the same.

Proof. Apply $(\mathscr{K})$ to the case $\alpha=\Delta_{1}, c_{1}=A_{1}, c_{2}=B_{12}, c_{3}=B_{13}$, $c_{4}=B_{14}, c_{5}=B_{15}, c_{6}=B_{16}$. Then

$$
\alpha_{12}=\Delta_{27}^{1}, \alpha_{13}=\Delta_{37}^{1}, \alpha_{14}=\Delta_{47}^{1}, \alpha_{23}=\Delta_{237}^{1}, \alpha_{24}=\Delta_{247}^{1}, \alpha_{34}=\Delta_{347}^{1} .
$$


Then we have

$$
\begin{array}{lllllll}
\Delta_{27}^{1} & \Delta_{37}^{1} & \Delta_{47}^{1} & B_{15} & B_{16} & B_{1}^{7} \\
\Delta_{27}^{1} & \Delta_{237}^{1} & \Delta_{247}^{1} & B_{15} & B_{16} & A_{27} \\
\Delta_{37}^{1} & \Delta_{237}^{1} & \Delta_{347}^{1} & B_{15} & B_{16} & A_{37} \\
\Delta_{47}^{1} & \Delta_{247}^{1} & \Delta_{347}^{1} & B_{15} & B_{16} & A_{47} .
\end{array}
$$

The line common to the four reguli on the left is the second transversal other than $\Delta_{347}^{1}$ of $B_{15}, B_{16}, A_{37}, A_{47}$ and is therefore $\Gamma_{156}^{7}$. So $\Gamma_{156}^{7}$ meets $B_{1}^{7}$ and similarly $B_{5}^{7}, B_{6}^{7}$. In the same way, $B_{1}^{7}$ and $B_{6}^{7}$ both meet $\Gamma_{126}, \Gamma_{136}, \Gamma_{146}$. If $B_{1}^{7} \neq B_{6}^{7}$, then

$$
\mathscr{R}\left(B_{1}^{7} B_{6}^{7} B_{16}\right) \Rightarrow \mathscr{R}\left(\Gamma_{126}^{7} \Gamma_{136}^{7} \Gamma_{146}^{7} \Gamma_{156}^{7}\right) \Rightarrow \mathscr{R}\left(A_{27} A_{37} A_{47} A_{57}\right),
$$

which is a contradiction. So $B_{i}^{7}=B_{6}^{7}$ and similarly all six lines $B_{i}^{7}$ are the same.

Write $B_{7}$ for $B_{i}^{7}$. Thus there are seven lines $B_{i}$ and each $B_{i}$ meets the twenty lines $\Gamma_{j k l}^{i}$. Further, $\Delta_{j k}^{i}$ meets $B_{j}$. There are 42 double-sixes like

$$
\begin{array}{llllll}
B_{2} & B_{13} & B_{14} & B_{15} & B_{16} & B_{17} \\
\Delta_{1} & \Delta_{23}^{1} & \Delta_{24}^{1} & \Delta_{25}^{1} & \Delta_{26}^{1} & \Delta_{27}^{1} .
\end{array}
$$

$S_{12}$. Now, constructing the double-six on $\Gamma_{23}^{1}$ and $B_{23}, A_{14}, A_{15}, A_{16}, A_{17}$, we obtain

$$
\begin{array}{llllll}
B_{23} & A_{14} & A_{15} & A_{16} & A_{17} & B_{1} \\
\Gamma_{1} & \Gamma_{234}^{1} & \Gamma_{235}^{1} & \Gamma_{236}^{1} & \Gamma_{237}^{1} & \Gamma_{23}^{1} .
\end{array}
$$

So $B_{1}$ meets $\Gamma_{1}$ giving the seventh line apart from the six lines $A_{1 i}(i=2, \ldots, 7)$ to meet $\Gamma_{1}$.

$S_{13}$. Continuing the construction of Grace's figure on $\Delta_{1}$ from $S_{11}$, apply $(\mathscr{K})$ to the case $\alpha=\Delta_{1}, c_{1}=B_{12}, c_{2}=B_{13}, c_{3}=B_{14}, c_{4}=B_{15}, c_{5}=B_{16}$, $c_{6}=B_{17}$. Then

$$
\alpha_{12}=\Delta_{23}^{1}, \alpha_{13}=\Delta_{24}^{1}, \alpha_{14}=\Delta_{25}^{1}, \alpha_{23}=\Delta_{34}^{1}, \alpha_{24}=\Delta_{35}^{1}, \alpha_{34}=\Delta_{45}^{1} .
$$

Therefore, we have

$$
\begin{array}{lllllll}
\Delta_{23}^{1} & \Delta_{24}^{1} & \Delta_{25}^{1} & B_{16} & B_{17} & B_{2} \\
\Delta_{23}^{1} & \Delta_{34}^{1} & \Delta_{35}^{1} & B_{16} & B_{17} & B_{3} \\
\Delta_{24}^{1} & \Delta_{34}^{1} & \Delta_{45}^{1} & B_{16} & B_{17} & B_{4}^{\top} \\
\Delta_{25}^{1} & \Delta_{35}^{1} & \Delta_{45}^{1} & B_{16} & B_{17} & B_{5} .
\end{array}
$$

The reguli on the left have a common line $\gamma_{67}^{1}$, which meets $B_{16}, B_{17}, B_{2}, B_{3}$, $B_{4}, B_{5}$. 


\section{A CONFIGURATION OF LINES IN THREE DIMENSIONS 115}

No five of the lines $B_{1}, B_{2}, B_{3}, B_{4}, B_{67}$ lie in a regulus, since

$$
\mathscr{R}\left(B_{1} B_{2} B_{3} B_{4}\right) \Rightarrow \mathscr{R}\left(\gamma_{26}^{7} \gamma_{36}^{7} \gamma_{46}^{7} \gamma_{56}^{7}\right) \Rightarrow \mathscr{R}\left(B_{1} B_{2} B_{3} B_{67}\right)
$$

and since

$$
\mathscr{R}\left(B_{1} B_{2} B_{3} B_{67}\right) \Rightarrow \mathscr{R}\left(\Delta_{12}^{6} \Delta_{13}^{6} \Delta_{23}^{6} \Delta_{12}^{7} \Delta_{13}^{7} \Delta_{23}^{7}\right) \Rightarrow \mathscr{R}\left(B_{1} B_{47} B_{57} B_{67}\right),
$$

contradicting the existence of a double-six like that in $S_{11}$.

Therefore, using the lines $\gamma_{j k}^{i}$, we have the double-sixes

and

$$
\begin{array}{ccccccc}
D: & B_{1} & B_{2} & B_{3} & B_{4} & B_{5} & B_{67} \\
& \gamma_{16}^{7} & \gamma_{26}^{7} & \gamma_{36}^{7} & \gamma_{46}^{7} & \gamma_{56}^{7} & \Gamma_{6}^{7},
\end{array}
$$

$$
\begin{array}{ccccccc}
D^{\prime}: & B_{1} & B_{2} & B_{3} & B_{4} & B_{6} & B_{57} \\
& \gamma_{15}^{7} & \gamma_{25}^{7} & \gamma_{35}^{7} & \gamma_{45}^{7} & \gamma_{56}^{7} & \Gamma_{5}^{7} .
\end{array}
$$

Theorem. All six lines $\Gamma_{i}^{7}$ are the same.

Proof. $\Gamma_{6}^{7} \neq \Gamma_{5}^{7} \Rightarrow \mathscr{R}\left(\gamma_{56}^{7} \Gamma_{6}^{7} \Gamma_{5}^{7}\right) \Rightarrow \mathscr{R}\left(B_{1} B_{2} B_{3} B_{4}\right)$, contradicting the existenceof $D$. So all six $\Gamma_{i}^{7}$ are the same and will be written $\Gamma^{7}$.

Theorem. All three lines $\gamma_{67}^{5}, \gamma_{57}^{6}, \gamma_{56}^{7}$ are the same.

Proof. $\Gamma^{7} \neq \gamma_{57}^{6}$ as $\Gamma^{7}$ meets $B_{5}$ and $\gamma_{57}^{6}$ does not. So

$$
\gamma_{56}^{7} \neq \gamma_{57}^{6} \Rightarrow \mathscr{R}\left(\Gamma^{7} \gamma_{57}^{6} \gamma_{56}^{7}\right) \Rightarrow \mathscr{R}\left(B_{1} B_{2} B_{3} B_{4}\right),
$$

again a contradiction. So $\gamma_{56}^{7}=\gamma_{57}^{6}=\gamma_{67}^{5}$ and will be written $\Gamma_{567} \cdot \Gamma_{567}$ meets $B_{1}, B_{2}, B_{3}, B_{4}, B_{56}, B_{57}, B_{67}$.

$S_{14}$. Theorem. All seven lines $\Gamma^{i}$ are the same.

Proof. $\Gamma^{7} \neq \Gamma_{567}$ and $\Gamma^{6} \neq \Gamma_{567}$ since both pairs occur in a double-six. So $\Gamma^{7} \neq \Gamma^{6} \Rightarrow \mathscr{R}\left(\Gamma^{7} \Gamma^{6} \Gamma_{567}\right) \Rightarrow \mathscr{R}\left(B_{1} B_{2} B_{3} B_{4}\right)$, a contradiction. So $\Gamma^{7}=\Gamma^{6}$ and similarly all seven $\Gamma^{i}$ are the same.

Write $\Gamma=\Gamma^{i}$. Then $\Gamma$ meets $B_{1}, B_{2}, B_{3}, B_{4}, B_{5}, B_{6}, B_{7}$.

\section{Description of the configuration}

We now have the complete figure of $576 \mathrm{~g}$-lines and 56 l-lines, where each g-line meets seven l-lines and each l-line meets 72 g-lines. Further, the figure can be constructed from any g-line and the seven l-lines which meet it.

A change in notation now leads to a great simplification as below:

$\begin{array}{lllllllll}\text { Old } & \Delta & \Delta_{i j k} & \Gamma_{j k}^{i} & \Delta_{j k}^{i} & \Gamma_{i j k} & \Gamma & A_{i} & B_{i} \\ \text { New } & \Delta_{8} & \Delta_{i j k}^{8} & \Gamma_{j k 8}^{i} & \Delta_{j k 8}^{i} & \Gamma_{i j k}^{8} & \Gamma_{8} & B_{i 8} & A_{i 8}\end{array}$

$\Delta_{i}, \Gamma_{i}, \Delta_{j k l}^{i}, \Gamma_{j k l}^{i}, A_{i j}, B_{i j}$ remain the same. 
Now there are

$\begin{array}{rll}8 & \text { lines } & \Delta_{i} \\ 8 & \text { lines } & \Gamma_{i} \\ 280 & \text { lines } & \Delta_{j k l}^{i} \\ 280 & \text { lines } & \Gamma_{j k l}^{i} \\ 28 & \text { lines } & A_{i j} \\ 28 & \text { lines } & B_{i j}\end{array}$

where the indices vary from 1 to 8 with no repetitions.

The intersections of the lines are as follows:

$$
\begin{array}{lll}
\Delta_{i} & \text { meets } & B_{i j} \\
\Delta_{j k l}^{i} & \text { meets } & A_{j k}, B_{i m} \\
\Gamma_{j k l}^{i} & \text { meets } & A_{i m}, B_{j k} \\
\Gamma_{i} & \text { meets } & A_{i j} .
\end{array}
$$

The $576 \mathrm{~g}$-lines fall naturally into 36 double-eights, one like

$$
\begin{array}{llllllll}
\Delta_{1} & \Delta_{2} & \Delta_{3} & \Delta_{4} & \Delta_{5} & \Delta_{6} & \Delta_{7} & \Delta_{8} \\
\Gamma_{1} & \Gamma_{2} & \Gamma_{3} & \Gamma_{4} & \Gamma_{5} & \Gamma_{6} & \Gamma_{7} & \Gamma_{8}
\end{array}
$$

and $\left(\begin{array}{l}8 \\ 4\end{array}\right) / 2=35$ like

$$
\begin{array}{llllllll}
\Delta_{234}^{1} & \Delta_{341}^{2} & \Delta_{412}^{3} & \Delta_{123}^{4} & \Delta_{678}^{5} & \Delta_{785}^{6} & \Delta_{856}^{7} & \Delta_{567}^{8} \\
\Gamma_{234}^{1} & \Gamma_{341}^{2} & \Gamma_{412}^{3} & \Gamma_{123}^{4} & \Gamma_{678}^{5} & \Gamma_{785}^{6} & \Gamma_{856}^{7} & \Gamma_{567}^{8}
\end{array}
$$

where the significance is quite unlike that of the double-six, but, for example, if the construction is begun with the line $\Delta_{8}$ and the seven l-lines which meet it, then $\Gamma_{1}, \ldots, \Gamma_{7}$ are the seven Grace lines obtained and $\Gamma_{8}$ is the completing line of the configuration.

Each g-line occurs in exactly one double-eight. The eight g-lines forming one-half of a double-eight have, as transversals, 28 of the 1-lines: the eight g-lines forming the other half have the other 281 -lines as transversals.

Since each g-lines meets seven I-lines, there are $576 \times 21 / 6=2016$ doublesixes. They are as follows:

$\begin{array}{llllllll}m_{23}^{1} & A_{23} & B_{14} & B_{15} & B_{16} & B_{17} & B_{18} & 8\left(\begin{array}{l}7 \\ 2\end{array}\right)=168 \\ & \Delta_{1} & \Delta_{234}^{1} & \Delta_{235}^{1} & \Delta_{236}^{1} & \Delta_{237}^{1} & \Delta_{238}^{1} & \\ n_{23}^{1} & B_{23} & A_{14} & A_{15} & A_{16} & A_{17} & A_{18} & 168 \\ & \Gamma_{1} & \Gamma_{234}^{1} & \Gamma_{235}^{1} & \Gamma_{236}^{1} & \Gamma_{237}^{1} & \Gamma_{238}^{1} & \end{array}$


A CONFIGURATION OF LINES IN THREE DIMENSIONS

$\begin{array}{llllllll}p_{55678}^{11234} & A_{12} & A_{13} & A_{14} & B_{56} & B_{57} & B_{58} & 8.7 .\left(\begin{array}{l}6 \\ 3\end{array}\right)=1120 \\ & \Delta_{134}^{5} & \Delta_{124}^{5} & \Delta_{123}^{5} & \Gamma_{578}^{1} & \Gamma_{568}^{1} & \Gamma_{567}^{1} & \\ q_{456}^{123} & A_{23} & A_{31} & A_{12} & B_{56} & B_{64} & B_{45} & \left(\begin{array}{l}8 \\ 3\end{array}\right)\left(\begin{array}{l}5 \\ 3\end{array}\right)=560 \\ & \Gamma_{456}^{1} & \Gamma_{456}^{2} & \Gamma_{456}^{3} & \Delta_{123}^{4} & \Delta_{123}^{5} & \Delta_{123}^{6} & \end{array}$

Each g-line lies in 21 double-sixes and each l-line lies in 2016.6/56 $=216$ double-sixes.

There is a key theorem for this configuration which deals with five, six and seven skew lines having a transversal. Given $a$ line $b$ with $n$ transversals $a_{1}, \ldots, a_{n}$, the locus of points $P$ such that the $n+1$ planes $P b, P a_{1}, \ldots, P a_{n}$ touch a quadric is a cubic surface, a twisted cubic or a single point $P$ according as $n$ is five, six or seven, Baker (1), p. 195. Dually, the locus of planes $\pi$ such that the $n+1$ points $\pi . b, \pi \cdot a_{1}, \ldots, \pi . a_{n}$ lie on a conic is a cubic envelope, a cubic developable or a single plane according as $n$ is five, six or seven. Passing from five to six, six cubic surfaces all containing the twisted cubic are obtained: passing from six to seven, seven twisted cubics are obtained all containing the point.

For $n=7$, there are other naturally associated algebraic varieties. A quartic surface is determined by 34 conditions. For $b$ and $a_{1}, \ldots, a_{7}$ to lie on a quartic surface, there are $5+7 \cdot 4=33$ conditions. So there is a pencil of such surfaces through the eight lines. Two of these are special: firstly, one may require that the point $P$ obtained above lies on the surface; secondly, one may require that $b$ is a double line of the surface. Either condition gives a unique quartic surface. The former surface also contains the seven twisted cubics, each of which has six of the $a_{i}$ as chords. Both surfaces are described in (9).

Beginning with $\Gamma_{1}$ meeting $A_{12}, A_{13}, A_{14}, A_{15}, A_{16}, A_{17}$, six double-sixes are obtained with the completing lines $B_{28}, B_{38}, B_{48}, B_{58}, B_{68}, B_{78}$. These twelve l-lines are chords of a twisted cubic $t_{1}^{8}$. Thus there are 56 cubics $t_{i}^{j}$ with chords $A_{i k}, B_{j k}(k \neq i, j)$. From $\Gamma_{1}$ and the seven lines $A_{12}, \ldots, A_{18}$, the seven cubics $t_{1}^{i}(i \neq 1)$ are obtained, which are concurrent at the point $P_{1}$. Thus there are eight points $P_{i}$ and eight points $Q_{i}$, where $Q_{1}$, for example, is the meet of $t_{2}^{1}, \ldots, t_{8}^{1}$.

Beginning with $\Delta_{234}^{1}$ meeting $A_{23}, A_{24}, A_{34}, B_{15}, B_{16}, B_{17}$, six doublesixes with completing lines $A_{28}, A_{38}, A_{48}, B_{56}, B_{57}, B_{67}$ are obtained. These twelve lines are chords of a twisted cubic $t_{2348}$. Thus there are $\left(\begin{array}{l}8 \\ 4\end{array}\right)=70$ cubics $t_{i j k l}$ with chords $A_{i j}, B_{m n}(m, n \neq i, j, k, l)$. This gives 126 cubics in all. From sixes of the seven l-lines meeting $\Delta_{234}^{1}$, the seven cubics

$$
t_{2345}, t_{2346}, t_{2347}, t_{2348}, t_{2}^{1}, t_{3}^{1}, t_{4}^{1}
$$

are obtained and these meet at $Q_{1}$. Similarly, from the seven l-lines meeting 
$\Gamma_{234}^{1}$, the seven cubics $t_{1567}, t_{1568}, t_{1578}, t_{1678}, t_{1}^{2}, t_{1}^{3}, t_{1}^{4}$, are obtained and they meet at $P_{1}$.

Theorem. All 126 cubics $t_{i}^{j}, t_{i j k l}$ have a point $P$ in common.

Proof. From above, $t_{i}^{j}$ contains $P_{i}, Q_{j} ; t_{i j k l}$ contains $P_{i}, Q_{m}$. The two cubics $t_{1567}$ and $t_{1568}$ have the six chords $A_{15}, A_{16}, A_{56}, B_{23}, B_{24}, B_{34}$ in common and so at most one point in common. Thus

$$
t_{1567} \cdot t_{1568}=P_{1}=P_{5}=P_{6}=Q_{2}=Q_{3}=Q_{4} .
$$

Hence all $P_{i}$ and $Q_{j}$ are the same point, which will be called $P$. So all 126 cubics have the point $P$ in common.

Dually there are 126 cubic developables, all of which have a plane $\pi$ in common. $\pi$ meets each g-line and its seven transversals in the eight points of a conic. The eight planes joining $P$ to a g-line and its seven transversals touch a quadric cone with vertex $P$.

If a cubic surface contains five chords of a twisted cubic, then the surface contains the curve. $P$ lies on each of the 2016 cubic surfaces (containing the double-sixes), each of which contains two of the 126 twisted cubics. For example, $m_{23}^{1}$ contains the 1-lines $A_{23}, B_{14}, B_{15}, B_{16}, B_{17}, B_{18}$ and therefore just the two cubics $t_{2}^{1}, t_{3}^{1}$. So each cubic curve lies on $2016 \times 2 / 126=32$ surfaces. For example, $t_{1}^{2}$ lies on six surfaces $m_{1 i}^{2}$, six surfaces $n_{2 i}^{1}$ and twenty surfaces $p_{22 l m n}^{11 i j k}$.

If a twisted cubic has thirteen points in common with a quartic surface, the curve lies on the surface. So, for any g-line, there exists a unique quartic surface containing it, the seven 1-lines meeting it and the seven twisted cubics with sixes of the seven lines as chords. Thus the number of such surfaces through one of the 126 cubic curves is $576 \times 7 / 126=32$.

One final figure is the number of twisted cubics which have a given 1-line as chord, viz. $126 \times 12 / 56=27$.

Therefore, to summarise the numerical properties of the figure, there are the following tactical configurations:

$\begin{array}{ll}\text { g-lines, l-lines } & (576,7 ; 56,72) \\ \text { cubic curves, l-lines } & (126,12 ; 56,27) \\ \text { cubic surfaces, g-lines } & (2016,6 ; 576,21) \\ \text { cubic surfaces, l-lines } & (2016,6 ; 56,216) \\ \text { cubic surfaces, cubic curves } & (2016,2 ; 126,32) \\ \text { quartic surfaces, g-lines } & (576,1 ; 576,1) \\ \text { quartic surfaces, 1-lines } & (576,7 ; 56,72) \\ \text { quartic surfaces, cubic curves } & (576,7 ; 126,32) \\ \text { quartic surfaces, cubic surfaces } & (576,21 ; 2016,6) .\end{array}$

In the case of the last tactical configuration, for each quartic surface there are 21 cubic surfaces, which meet it in a degenerate curve of order 12 consisting 
of a g-line, five 1-lines and two twisted cubics; for each cubic surface, there are 6 quartic surfaces meeting it in such a curve.

If, in the construction of the configuration, $A_{7}$ is taken to be a chord of the unique twisted cubic $t$ with $A_{1}, \ldots, A_{6}$ as chords, then all 126 twisted cubics $t_{i}^{j}, t_{i j k l}$ are the same, viz. $t$, and all 56 l-lines $A_{i j}, B_{i j}$ are chords of $t$. Also, there is no special point $P$.

\section{Groups of the configuration}

From five skew lines with a transversal, the 27 lines of a cubic surface are obtained: they form 36 double-sixes. The substitutions which preserve the configuration are as follows: any two lines of one half of a double-six may be interchanged; the two halves of a double-six may be interchanged; any double-six may be transformed into any other. Thus the group, $G_{5}$, of substitutions of the twenty-seven lines has order $6 ! \times 2 \times 36=51,840$ and is well-known.

From six skew lines with a transversal, the 44 lines of Grace's extension are derived. They form 32 double-sixes with halves as follows:

$\begin{array}{rrrrrrrr}6 & \text { like } & d_{1} & c_{2} & c_{3} & c_{4} & c_{5} & c_{6} \\ 6 & \text { like } & c_{1} & d_{2} & d_{3} & d_{4} & d_{5} & d_{6} \\ 20 & \text { like } & c_{1} & c_{2} & c_{3} & d_{4} & d_{5} & d_{6} .\end{array}$

The generators of the group, $G_{6}$, of the configuration are

$$
\left(c_{i} c_{j}\right)\left(d_{i} d_{j}\right), \quad\left(c_{i} d_{i}\right)\left(c_{j} d_{j}\right), \quad i, j=1, \ldots, 6 ;
$$

i.e. these generate all permutations of the lines of any half of a double-six and all interchanges of any two halves. So $G_{6}$ has order $32 \times 6 !=23,040$.

Let $M$ and $N$ be the following subgroups of $G_{6}: M=\left\langle\left\{\left(c_{i} c_{j}\right)\left(d_{i} d_{j}\right)\right\}\right\rangle$ and $N=\left\langle\left\{\left(c_{i} d_{i}\right)\left(c_{j} d_{j}\right)\right\}\right\rangle . \quad M$ is isomorphic to $S_{6} . \quad N$ is abelian and has order 32 ; so $N$ is an elementary abelian group of order 32 and isomorphic to

$$
C_{2} \times C_{2} \times C_{2} \times C_{2} \times C_{2} \text {, }
$$

where $C_{2}$ is cyclic of order 2. Further $N$ is a normal subgroup of $G_{6}$. Therefore $G_{6}=M N$ and is a split extension (or semi-direct product) of $N$ by $M$.

The order of the group, $G_{7}$, of the configuration that has been derived from seven skew lines with a transversal is best seen from the double-eights. Any two lines in the same row of one may be interchanged; the two rows of a double-eight may be interchanged; and any double-eight may be changed into any other. So the order of $G_{7}$ is

$$
8 ! \times 2 \times 36=2,903,040 \text {. }
$$

The groups $G_{5}, G_{6}, G_{7}$ are related to the semi-simple Lie algebras $E_{6}, D_{6}, E_{7}$, Bourbaki (2), pp. 256-266, and to the reflexion groups whose fundamental 
regions are the spherical simplexes $E_{6}, B_{6}, E_{7}$, Coxeter (5), ch. 11. The corresponding Dynkin graphs are

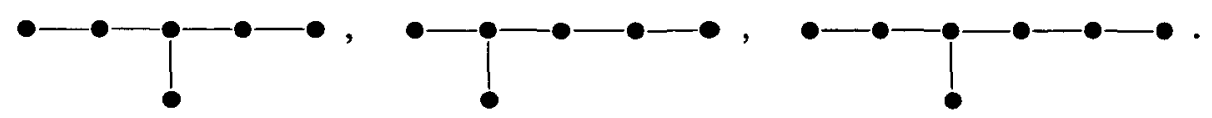

$G_{5}$ and $G_{6}$ are subgroups of $G_{7}$ of indices 56 and 126 respectively.

The group $G_{7}$ has an element $\gamma$ of order two, where

$$
\gamma=\prod_{i, j, k, l, m, n, p}\left(A_{i j} B_{i j}\right)\left(\Delta_{k} \Gamma_{k}\right)\left(\Delta_{l m n}^{p} \Gamma_{l m n}^{p}\right)
$$

i.e. $\gamma$ interchanges $A_{i j}$ and $B_{i j}, \Delta_{k}$ and $\Gamma_{k}, \Delta_{l m n}^{p}$ and $\Gamma_{l m n}^{p}$ for all values of the indices.

There exists a polarity which interchanges the lines $a_{i}$ and $b_{i}(i=1, \ldots, 6)$ of a double-six. Further, there exists a polarity which interchanges the lines $\alpha$ and $\beta, c_{i}$ and $d_{i}, \alpha_{j k}$ and $\beta_{j k}(i, j, k=1, \ldots, 6)$ of Grace's extension of the double-six. So it seems natural to postulate the following.

Conjecture. There exists a polarity which induces $\gamma$ and interchanges the point $P$ and the plane $\pi$.

The group $G_{7}$ has a subgroup of index two which is isomorphic to the group of the 28 bitangents of a non-singular plane quartic curve, (4). In fact, it will be shown that the configuration obtained has-modulo the involution $\gamma$-corresponding geometrical properties to those of the 28 bitangents. Thus the group of the bitangents is isomorphic to $G_{7} \mid\langle\gamma\rangle$.

\section{The correspondence between the configuration and the bitangents of a non- singular plane quartic curve}

Firstly, it is necessary to give a brief review of the properties of the 28 bitangents of a non-singular plane quartic curve $Q$; cf. Salmon (12), p. 223.

Let the bitangents be denoted by $T_{i j}(i, j=1, \ldots, 8, i<j)$. Then, given any pair of bitangents, five other pairs are uniquely determined so that any two of the six pairs have their eight points of contact with $Q$ on a conic. Such a set of 12 lines is called a Steiner set. There are 63 Steiner sets-28 like $\left\{T_{1 i}, T_{2 i} \mid i=3, \ldots, 8\right\}$ and 35 like

$$
\left\{T_{12}, T_{34} ; T_{13}, T_{24} ; T_{14}, T_{23} ; T_{56}, T_{78} ; T_{57}, T_{68} ; T_{58}, T_{67}\right\}
$$

There exist sets of seven bitangents, called Aronhold sets, such that no three of the seven have their six points of contact on a conic. There are 288 Aronhold sets-8 like $\left\{T_{1 i} \mid i=2, \ldots, 8\right\}$ and 280 like $\left\{T_{12}, T_{13}, T_{14}, T_{15}, T_{67}, T_{68}, T_{78}\right\}$

There are 36 ways in which the 28 bitangents can be arranged (each appearing twice) as the elements of an $8 \times 8$ symmetric matrix excluding the main diagonal such that each row and column is an Aronhold set. This is a Hesse arrangement. It is, in fact, the basis for the notation $T_{i j}$ for the bitangents. 
Thus the 288 Aronhold sets fall naturally by eights into the 36 Hesse arrangements.

Any five bitangents of an Aronhold set determine a sixth such that their twelve points of contact lie on a cubic curve: the six lines also touch a conic. There are 1008 of these hexads-168 like $\left\{T_{23}, T_{14}, T_{15}, T_{16}, T_{17}, T_{18}\right\}, 560$ like $\left\{T_{12}, T_{13}, T_{14}, T_{56}, T_{57}, T_{58}\right\}$ and 280 like $\left\{T_{12}, T_{13}, T_{23}, T_{45}, T_{46}, T_{56}\right\}$.

Finally, given seven general lines in the plane, there exists a unique quartic curve having these lines as bitangents. The remaining 21 bitangents can be constructed linearly from the initial seven.

The correspondence between the bitangents of $Q$ and our configuration is then as follows.

Number Plane object

Space object

36 Hesse arrangement of Double-eight of g-lines the 28 bitangents

63 Steiner set of 12 lines Pair of twisted cubics each with 12 chords

288 Aronhold set of 7 lines Pair of g-lines each with 7 transversal l-lines

1008 Cubic curve and set of Pair of cubic surfaces each containing 6 lines

6 l-lines

The pairs of space objects which are the same modulo $\gamma$ are as follows:

$$
\begin{aligned}
& \text { cubics } \quad\left\{\begin{array}{lll}
t_{i}^{j} & \text { with chords } A_{i k}, B_{j k} & (k \neq i, j) \\
t_{j}^{i} & \text { with chords } A_{j k}, B_{i k} & (k \neq i, j)
\end{array}\right. \\
& \text { cubics } \quad \begin{cases}t_{i j k l} \text { with chords } A_{i j}, B_{m n} & (m, n \neq i, j, k, l) \\
t_{m n p q} \text { with chords } A_{m n}, B_{i j} & (i, j \neq m, n, p, q)\end{cases} \\
& \text { g-lines } \quad\left\{\begin{array}{llll}
\Delta_{i} & \text { with transversals } & B_{i j} & (j \neq i) \\
\Gamma_{i} & \text { with transversals } & A_{i j} & (j \neq i)
\end{array}\right. \\
& \text { g-lines } \quad\left\{\begin{array}{llll}
\Delta_{j k l}^{i} & \text { with transversals } & A_{j k}, B_{i m} & (m \neq i, j, k, l) \\
\Gamma_{j k l}^{i} & \text { with transversals } & A_{i m}, B_{j k} & (m \neq i, j, k, l)
\end{array}\right. \\
& \text { cubic surfaces }\left\{\begin{array}{llll}
m_{j k}^{i} & \text { containing l-lines } & A_{j k}, B_{i l} & (l \neq i, j, k) \\
n_{j k}^{i} & \text { containing l-lines } & A_{i l}, B_{j k} & (l \neq i, j, k)
\end{array}\right. \\
& \text { cubic surfaces }\left\{\begin{array}{l}
p_{m m n p q}^{i j k k l} \text { containing l-lines } A_{i j}, B_{m n} \\
p_{i j i k l}^{m m n p q} \text { containing l-lines } A_{m n}, B_{i j}
\end{array}\right. \\
& \text { cubic surfaces }\left\{\begin{array}{lll}
q_{l m n}^{i j k} & \text { containing l-lines } & A_{i j}, B_{l m} \\
q_{i j k}^{l m n} & \text { containing l-lines } & A_{l m}, B_{i j} .
\end{array}\right. \\
& \text { E.M.S. -H }
\end{aligned}
$$


It seems possible that the correspondence could be made more precise. If the 561 -lines are projected from $P$ on to $\pi$, the 56 lines $P l \cap \pi$ are obtained, which touch 576 conics in sets of seven. Dually, the 56 points of intersection $\ln \pi$ lie in sevens on 576 conics. It seems doubtful that these 56 points could be the points of contact of 28 bitangents of a quartic curve, since they do not lie suitably in eights on conics.

\section{Conclusion}

The complete configuration of 632 lines derived from seven skew lines with a transversal has been obtained. Although we know that the seven Grace lines $\Gamma_{1}, \ldots, \Gamma_{7}$ do not in general have a transversal, $\S 1$, it has not been shown without using a computed example. If one supposed that the seven Grace lines did have a transversal, then the eight g-lines of any row of a double-eight would have a transversal. It is therefore possible that, to understand the above configuration completely, it is necessary to investigate the configuration obtainable from eight skew lines with a transversal. For this case, however, there does not seem to be an associated irreducible variety, as was the case for five, six and seven lines.

From Longuet-Higgins's examples (11), it seems highly probable that the seven Grace lines always belong to a linear complex. This has two consequences. Firstly, it means that the eight g-lines of any row of a double-eight belong to a linear complex, thus giving 72 linear complexes in all. Secondly, by a theorem of Todd (13), p. 63, it means that any seven g-lines belonging to one half of a double-eight lie on a quartic surface, thus associating another set of 576 quartic surfaces with our figure.

\section{REFERENCES}

(1) H. F. BAKER, Principles of Geometry, Vol. III (Cambridge University Press, 1923).

(2) N. Bourbaki, Groupes et Algèbres de Lie, Ch. 4, 5, and 6 (Hermann, Paris, 1968).

(3) L. M. Brown, A configuration of points and spheres in four-dimensional space, Proc. Roy. Soc. Edinburgh Sect. A 64 (1955), 145-149.

(4) H. S. M. COXETER, Math. Reviews 17 (1956), 886.

(5) H. S. M. CoXeter, Regular Polytopes (Macmillan, 1963).

(6) J. H. Grace, Circles, spheres and linear complexes, Trans. Cambridge Philos. Soc. 16 (1898), 153-190.

(7) J. W. P. HiRschFeld, Classical configurations over finite fields: I. The doublesix and the cubic surface with 27 lines, Rend. Mat. e Appl. 26 (1967), 115-152.

(8) J. W. P. HIRSCHFELD, Classical configurations over finite fields: II. Grace's extension of the double-six, Rend. Mat. e Appl. 26 (1967), 349-374. 
A CONFIGURATION OF LINES IN THREE DIMENSIONS 123

(9) J. W. P. HIRSCHFELD, A projective configuration, Computational Problems in Abstract Algebra (Pergamon, 1970), 321-324.

(10) T. KuвотA, On double sixers, Sci. Rep. Tôhuku Univ. 6 (1917), 89-94.

(11) M. S. Longuet-Higgrns, A numerical disproof of a conjecture in projective geometry, Proc. Roy. Soc. Ser. A 323 (1971), 443-453.

(12) G. Salmon, Higher Plane Curves (3rd ed., Dublin, 1879).

(13) J. A. TodD, Configurations defined by six lines in space of three dimensions, Proc. Cambridge Philos. Soc. 29 (1933), 52-68.

(14) T. L. Wren, Some applications of the two-three birational space transformation. Proc. London Math. Soc. 15 (1916), 144-165.

UNIVERSITY OF SUSSEX

ENGLAND 